\title{
21st Century Approach: Using data and novel technologies to address the opioid crisis
}

\section{Eric Yazel ${ }^{2}$, Jessica B. Dennison ${ }^{2}$, Crystal L. Henderson ${ }^{1}$}

${ }^{1}$ IU Richard M. Fairbanks School of Public Health, Indianapolis, Indiana, United States, ${ }^{2}$ Clark Memorial Hospital, Jeffersonville, Indiana, United States Objective

To use novel technologies to develop a rapid response framework to reach opioid overdose patients in an area which is challenging from both a geography and population distribution standpoint.

\section{Introduction}

Clark County, Indiana is geographically located in between the urban area of Louisville, Kentucky and Scott County, Indiana. Scott County is the site for the largest HIV outbreak in the history of the United States, directly related to high rates of IV drug abuse. The unique geographic location of Clark County in combination with the recent HIV and Hepatitis C outbreaks in Clark and neighboring counties has greatly informed the development of an effective response to overdoses and the opioid epidemic in general. Furthermore, Clark County has a unique population distribution, with a population of over 125,000 and a land area of over 300 square miles. Despite this large area, over $80 \%$ of the population lives within 9 miles of the southern border of the county. This leads to a mix of both urban and rural challenges. There are several areas of the county that have greater than 15 minute emergency response times, which is often the difference between life and death in an overdose situation. These factors led to the development of the Clark County Rapid Response Project. The rapid response project is a community-based, multidisciplinary framework to address the opioid addicted patient, from initial use to successful recovery. The project uses data driven technology to initiate the care of opiate overdose patients and administer lifesaving interventions.

\section{Methods}

Clark County has partnered with the Indiana State Department of Health utilizing the early notification system that monitors statewide overdose activity. Once an alert is sent out, the response involves the use of two early notification systems. Everbridge is a one touch notification system that allows rapid dissemination of information to various community partners to allow them to initiate the appropriate response. Pulse Point is a smart phone application that allows CPR and trained community laypeople to respond to a cardiac arrest or overdose patient in a public place. It provides directions to the patient as well as to the nearest AED. Clark County has also simultaneously instituted a county-wide CPR training initiative and offered Narcan training as well. This is a major paradigm shift, as prior methods of deployment of trained laypeople essentially relied on the chance that an overdose will be reached by a first responder.

\section{Results}

Everbridge has allowed for the rapid notification of county entities and deployment of resources to overdose 'hot spot' areas. The Pulse Point initiative has dramatically increased the number of CPR and Narcan trained responders and provided means of delivering them to the appropriate patient population in a timely manner. Both these technologies have dramatically increased the delivery of resources to the overdose patient and decreased response times to the delivery of care. Conclusions Using data driven technology to inform how Clark County Health Department and first responders collectively address the opioid crisis is a novel approach. Since January 2018, Clark County Health Department has used ESSENCE (Electronic Surveillance System for the Early Notification of Community- based Epidemics) to determine where and when an increase of drug overdose activity is occurring throughout the county. This affords county health officials the ability to inform in "near real-time" first responders, the emergency department and other community stakeholders, relevant information thus allowing for the rapid deployment of county resources to the areas most affected. Our collective efforts to save lives is further enhanced by the county using of novel technologies like Pulse Point which is used to deploy both CPR and Narcan trained laypersons directly to sites in the community where overdoses are occurring. In a community, which is in large part considered rural and, in many places, has a greater than 15 minute emergency response time, using Pulse Point and Everbridge technologies has uniquely positioned Clark County to be on the cutting edge of 
saving lives as we leverage data and technology to address the opioid epidemic in our communities. This has markedly improved access to treatment and response times to overdose patients in Clark County, Indiana.

\section{Acknowledgement}

The authors of this abstract would like to acknowledge the Indiana State Department of Health, Lifespring Health Services, Clark Memorial Hospital, and the Community Foundation of Southern Indiana. Each institution is a stakeholder and representatives from each are working alongside us as we strive to disrupt and address the opioid crisis in meaningful and tangible ways. 